\title{
Primary healthcare midwives' experiences of caring for parents who have suffered an involuntary pregnancy loss: a phenomenological hermeneutic study
}

\author{
Sara Fernández-Basanta ${ }^{\mathrm{a}}$, Carmen Coronado ${ }^{\mathrm{b}}$, Terese Bondas ${ }^{\mathrm{c}}$, María-Jesús Movilla- \\ Fernández \\ ${ }^{a}$ Research group GRINCAR, Department of Health Sciences, Faculty of Nursing and Podiatry, University of A \\ Coruña, Naturalista López Seoane s/n, 15471 Ferrol, Spain \\ ${ }^{b}$ Associate professor. Research group GRINCAR, Department of Health Sciences, Faculty of Nursing and Podiatry, \\ University of A Coruña, Naturalista López Seoane s/n, 15471 Ferrol, Spain. \\ ${ }^{c}$ Professor (Full) and Leader of 'Childbearing - the Qualitative Research Network'. Faculty of Health Sciences, \\ University of Stavanger, PO Box 8600, Forus, Stavanger, Norway. \\ ${ }^{d}$ Associate professor. Research group GRINCAR, Department of Health Sciences, Faculty of Nursing and Podiatry, \\ University of A Coruña, Naturalista López Seoane s/n, 15471 Ferrol, Spain.
}

\begin{abstract}
Objective. To illuminate the experiences of primary healthcare midwives who care for parents who have suffered an involuntary pregnancy loss.

Design. The phenomenological hermeneutic approach developed by Lindseth and Norberg was used to carry out narrative interviews.

Setting and participants. A purposive sample of 11 public primary healthcare midwives from a municipality in northern Spain, was selected. The participants' ages ranged between 26 and 62 years, and they were all women.

Findings. Four main themes were identified: (1) handling adversity, (2) finding a motive to get involved, (3) providing care from the rear, and (4) avoiding emotional connections with the parents. For the midwives, caring for parents who had suffered an involuntary pregnancy loss meant leaving their own comfort zone and handling adversity. They described acting in different ways such as going beyond task-focused care, following their intuition or avoiding encounters with the parents.

Conclusions. More knowledge and preparation in terms of communication skills and bereavement is crucial for midwives in order to meet the needs of parents who have suffered an involuntary pregnancy loss. A caring organizational culture and supportive leadership will facilitate care continuity between specialized and primary healthcare and promote the welfare of midwives.
\end{abstract}

\section{Keywords}

Caring, Midwifery, Miscarriage, Primary Healthcare, Qualitative Research, Stillbirth 


\section{Introduction}

Involuntary pregnancy loss (IPL), including miscarriages and stillbirths, is a common occurrence, and, therefore, midwives have a high probability of encountering families who have experienced IPL in a current or previous pregnancy (Heazell et al., 2019). This loss can lead to a set of painful emotional reactions by the parents (Flenady et al., 2014). Previous research has focused mainly on the impact on women (Fernández-Basanta et al., 2019), whereas the experiences of men have been given little attention (McCreight, 2004).

The literature has highlighted the importance of the care provided by healthcare professionals in the management of these losses (Fernández-Basanta et al., 2020a). Midwives have a fundamental role in this care because of their frequent encounters with the parents (Ellis et al., 2016; Gold, 2007). However, knowledge, skills, and personal sacrifice are required in order to avoid delivering superficial and taskfocused care (Ellis et al., 2016; Martínez-Serrano et al., 2018). Generally, recommendations are to provide care soon after the loss occurs, but follow-up has not been addressed in depth (Peters et al., 2016).

In the Spanish primary healthcare system, midwives are those who follow up a pregnancy and, in many cases, are the professionals who refer the patient to the hospital when they suspect a miscarriage or stillbirth has occurred. Therefore, the midwives function as the link between the parents and the healthcare system. They are also the professionals that accompany the parents throughout the subsequent process of loss. Although nurses' and midwives' experiences of caring for parents who have suffered an IPL have been synthesised previously in the literature, little research has been conducted on the primary healthcare midwife's perspective (Fernández-Basanta et al., 2020b). Currently, few guidelines exist for this area of healthcare; therefore, the care provided is based on each midwife's own professional judgement (López García de Madinabeitia, 2011).

\section{Theoretical perspective}

This study employed the Caritative Caring Theory (Eriksson, 1994) to gain a better understanding of the complexity and wholeness of the experiences of primary healthcare midwives in the care of IPLs. This theory assumes that caring is the core of nursing. Caritative caring involves a caring encounter between the suffering human being and the caregiver that is based on their own experiences and wishes.

Ontologically, the human being is conceived as an indivisible entity that includes body, soul, and spirit. The ethos of care includes caritas, love, and charity; the respect of health professionals for the dignity of the person; and a striving for a genuine communion and understanding of the unique human being. Caritas represents the unconditional love that constitutes the motive for caring. Therefore, caritative care is based on the relationship between the person who needs and hopes for care and the person who provides the care, through genuine communion and understanding of the uniqueness of the human being.

Aim

To illuminate care for parents, who have suffered an IPL from the perspective of primary health care midwives. 


\section{Methods}

\section{Design}

This qualitative study used a phenomenological hermeneutic design that was inspired by Ricoeur (1976) and developed by Lindseth and Norberg (2004). Hermeneutic phenomenology is conceived of as the philosophy of understanding and the science of textual interpretation (Wiklund et al., 2002), which has been used successfully in research in midwifery and nursing. It is an appropriate approach to illuminate the phenomenon from an insider perspective of primary healthcare midwives. We wanted to focus on the understandable meaning of these experiences (Lindseth and Norberg, 2004).

Lincoln and Guba (1985) proposed the following trustworthiness criteria for carrying out naturalistic inquiries: 'credibility', 'confirmability', 'transferability', and 'dependability'. This study was carried out maintaining theoretical-epistemological adaptation and consistency. Credibility was ensured through the presentation of data according to the authors 'interpretations, along with the comments of the midwives. During the analysis, the researcher's notes (collected after the interviews) were used, analysis was triangulated by all the authors, and findings were offered the possibility of recognition and comments by the midwives. Furthermore, the researchers' reflections on their own positions on the topic and an audit trail that detailed the process of data analysis and interpretation of the data guaranteed confirmability. Transferability was facilitated through a description of the context and the participants. To enhance dependability, detailed descriptions of the study sample, data collection process, analysis and interpretation of the data, role of researchers, and study limitations are presented.

Analysis and interpretation of the data was triangulated by the authors to strengthen the interpretation by contesting and supplementing each other's readings. All authors were trained qualitative researchers who had experience working on the topic of IPL. Moreover, one of the authors has professional experience working in a gynaecological hospital and in primary healthcare encountering parents who have experienced IPLs.

The authors' preunderstandings to be addressed during the study were that the care provided after an IPL should be focused on the human being as a whole and continue from the hospital to primary care and that the midwife is who has the competencies to provide care and follow up to parents who suffer IPLs.

\section{Participants}

Eleven midwives working in 10 primary healthcare centres in Spain, voluntarily participated in the study. The number of potential participants from the 10 centres was 13. Two midwives did not participate (one due to family commitments and the other could not be reached). A purposive sample was performed, according to the study aim. In order to recruit participants, two authors (SFB and MJMF) attended the monthly meeting attended by all primary healthcare midwives. Details of the study were presented at the meeting and written information was given to the attendees. The inclusion criteria were being a primary healthcare midwife and having experience in providing care to parents who have suffered an IPL.

\section{Data collection}

According to Lindseth and Norberg (2004), narrative interviews are an appropriate method for disclosing the meaning of lived experience. Data were collected between February and April 2019 by SFB. Participants were able to choose the location for the interview. Most of the interviews were conducted in the midwives' offices at the healthcare centres. Two were conducted at the Faculty of Nursing and Podiatry at the University of $\mathrm{X}$. The interviews lasted 60 minutes on average. A guide based on a literature review and previous knowledge was used to conduct the interviews. All interviews commenced with the prompt 'Tell me about your experience of providing care for an IPL as a primary healthcare provider'. A question route with main topics based on literature research and previous knowledge was used (Table 1). Further questions that encouraged additional narration were asked. Field notes collected after the interviews were integrated into the transcripts to enrich the data. The interviews were conducted 
in Spanish or Galician and were tape-recorded and transcribed (by SFB). Sufficiency of data to enable the illumination of the phenomenon was reached, since the data provided enough information to reflect on and validate the naïve understanding.

Table 1. Narrative interview script.

\begin{tabular}{ll}
\hline Thematic field & Questions \\
\hline $\begin{array}{l}\text { Care provided for involuntary } \\
\text { pregnancy losses }\end{array}$ & How do you act when an involuntary pregnancy loss occurs? \\
& $\begin{array}{l}\text { How do you act with the father? } \\
\text { What difficulties do you experience regarding the care? } \\
\text { Training }\end{array}$ \\
& $\begin{array}{l}\text { Do you think that in your professional career these losses are addressed in education or } \\
\text { training? }\end{array}$ \\
& $\begin{array}{l}\text { How useful is the current training in imparting the knowledge and skills necessary to } \\
\text { provide care for and manage this situation? }\end{array}$ \\
& How do you feel when you face these situations? \\
Personal impact of involuntary & What impact does the care of these losses have on you? \\
pregnancy losses & How do you cope with caring for parents that have experienced an involuntary pregnancy \\
& loss?
\end{tabular}

\section{Ethical considerations}

Midwives were provided with verbal and written information on the study, and prior written informed consent was obtained from each participant. All midwives were informed about guaranteed confidentiality, the voluntariness of participation and the right to discontinue the interview at any time. The study obtained the approval of the Autonomous Committee of Research Ethics of Galicia (registration code 2015/232) and had the access permission by health area of X. The interviews were anonymized, and the audio recordings were destroyed after transcription.

\section{Data analysis}

The transcribed interviews were interpreted using a phenomenological hermeneutic method developed by Lindseth and Norberg (2004). The first step involved a naïve reading, in which three authors (SFB, CC, and MJMF) grasped the meaning as a whole and formulated a naïve understanding of the text. Next, a thematic structural analysis, which means the methodical instance of interpretation, was carried out by SFB. Any text relating to the experience of providing care to parents who have experienced IPLs was read through several times. Analytic questions were posed, and answers were searched for in the text. The text was divided into meaning units that were based on the aim of the study. The meaning units were condensed and abstracted to form preliminary subthemes and themes, which were compared with the naïve understanding for validation. The structural analysis was discussed in a dialectic movement between understanding and explanation until all the authors agreed. Finally, a comprehensive understanding was elaborated, based on the naïve understanding, structural analysis, study context, the researchers' preunderstandings, and theoretical perspectives. During this step, TB audited the analysis process and results.

The preunderstandings of the authors were discussed throughout the process in order to avoid distortion in the interpretation of the data. These processes were recorded in notes. To support the data analysis, grids created for this purpose were used. 


\section{Findings}

All 11 midwives were Spanish and trained in Spain. They all had experience in specialized and primary healthcare. The age range was 26 to 62 years, and all were women. Table 2 presents their sociodemographic characteristics.

Table 2. Sociodemographic characteristics of the participants

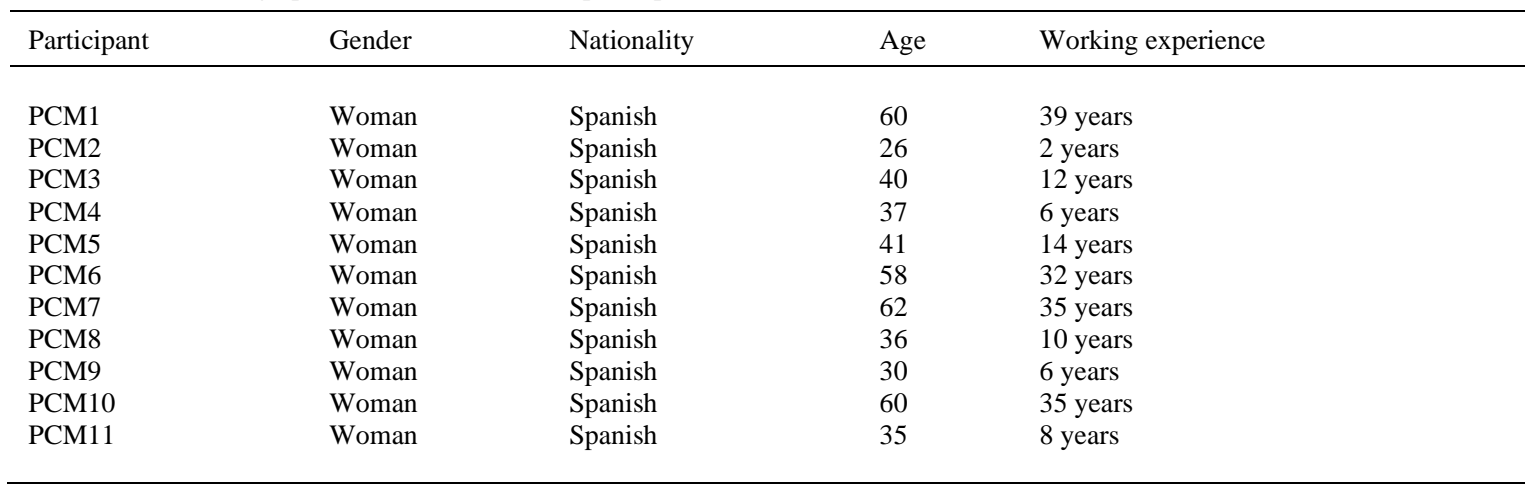

*Abbreviations: PCM (Primary Care Midwife)

Four main themes were identified: 'handling adversity', 'finding a motive to get involved', 'providing care from the rear', and 'avoiding emotional connections with the parents'.

\section{Naïve understanding}

Midwives reported that providing care to parents who have suffering an IPL involved leaving their own comfort zone and dealing with various forms of adversity, such as lack of training and knowledge, personal costs resulting from the provision of care, lack of organizational support, and lack of or little communication between specialised and primary healthcare providers. Under these circumstances, midwives had to look for reasons to go beyond task-focused care. The bond with the mother, coping strategies, a strong sense of professional responsibility, and having experienced the loss of a loved one were drivers. Care, therefore, meant that midwives got involved following their intuition, or that they avoided going beyond the provision of task-focused care because of their difficult encounters with the suffering parents.

\section{Structural analysis}

The structural analysis identified four themes of the lived experience of primary healthcare midwives who provide care to parents who have experienced an IPL (figure 1). 


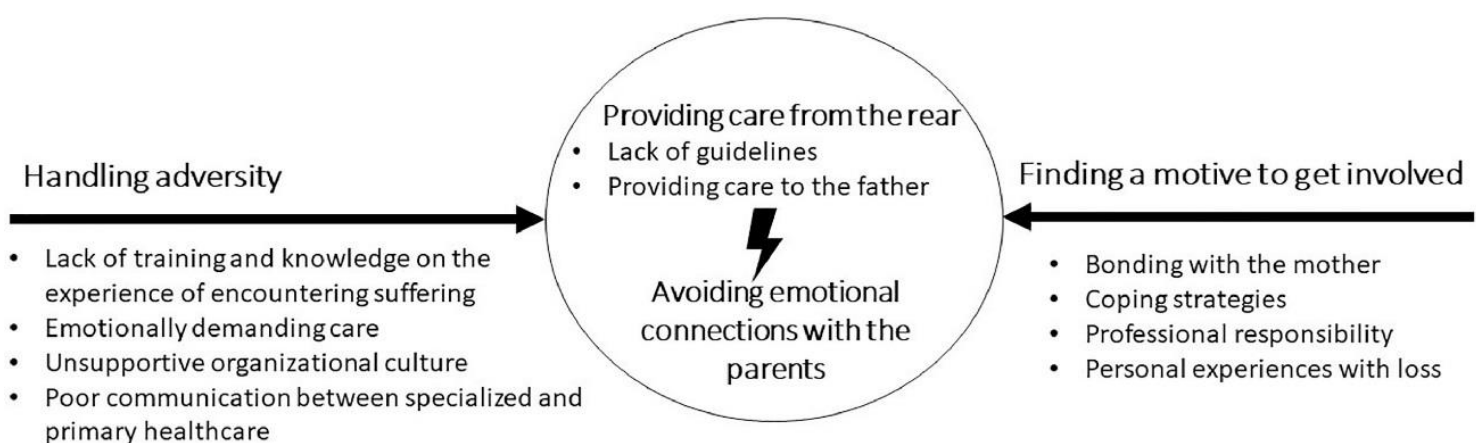

Fig. 1. Themes and subthemes of the lived experience of primary healthcare midwives who provide care to parents who have experienced IPL.

\section{Handling adversity}

Lack of training and knowledge on the experience of encountering suffering, emotionally demanding care, unsupportive organizational culture, and poor communication between specialized and primary healthcare were the difficulties that midwives had to deal with in the care of parents who have IPLs.

Lack of training and knowledge on the experience of encountering suffering

Midwives felt poorly prepared to care for the parents in the context of primary healthcare. Their training had not exposed them to these experiences, because their supervisors had wanted to protect them. Some commented that the lack of training reflects society's avoidance of topics related to death and suffering.

The limited training available did not provide enough knowledge and skills to manage the emotional demands of this type of care. Moreover, the training did not address the care provided in primary healthcare and it did not include the experiences of women following IPLs.

We are not trained on how to deal with those losses. We don't know how we can help the woman. A lot of training is missing. That is, how to manage them [the losses], and how to deal with them, and how to care for the couple. (PCM11)

Midwives supplemented their lack of training with private training, peer learning, and the knowledge and skills acquired from their personal and professional experiences of loss. This allowed them to understand how the woman could feel and improved communication and empathy with the parents. However, private training meant that midwives had to be trained outside working hours, incurred travel costs, and may not have been able to fully participate in the private training due to the difficulties in balancing work and family life.

If you have experienced something ... [personal experience provides you with] some tools to face it. On your own, not because you had training (PCM5)

As a result of lack of knowledge, midwives did not feel fully capable of leading in the provision of care and did not understand their role as the healthcare provider to the parents. Furthermore, at some point they experienced fear of failing in this role.

Emotionally demanding care

Caring for parents who have experienced an IPL entailed personal costs because of the emotional involvement with the parents. Being involved in this form of care was a challenge for midwives, because it forced them to leave their own comfort zone. Moreover, this care was in many ways the opposite of midwifery, which is the care of life. There could be questions of whether the care provided was adequate and the midwife may experience fear of legal repercussions in advanced losses. Nonetheless, some of 
them felt protected from legal reprisals, as primary healthcare providers. Metaphorically they feel that they are not in the front line of war, but in the rear.

I really like to listen, and I let them talk and I listen to them. But then that also makes you emotional. Then you have to know how to let go emotionally. (PCM4)

It was especially difficult for them to manage their emotions after being exposed to those of the parents. In primary healthcare, the suspicion of IPL after not finding the heartbeat is emotionally devastating. In this context, the link between the midwife and the woman was closer before the IPL, especially in advanced losses. This original closeness entails higher personal costs because the woman is more able to express her discontent. For example, for one midwife, it was difficult to feel the mother's rejection: But it was very painful. It was hard feeling her rejection (PCM7).

Unsupportive organizational culture

Midwives found that the healthcare system did not provide them with resources for managing the emotional impact of their work with the parents. Although many of the midwives mentioned that the workload in healthcare centres is no excuse for not going beyond task-focused care, some midwives reported that the limited time for consultations sometimes prevented them from being more involved with the couple.

I believe that healthcare professionals should take care of ourselves-the care of the healthcare professional. If you do not do it on your own, the system also does not worry that this therapy will be performed. (PCM3)

Poor communication between specialized and primary healthcare

Poor communication between specialized and primary care can cause a lack of continuity of care. For instance, on suspicion of a pregnancy loss, referred to the hospital for diagnosis, women may be sitting in waiting rooms with other pregnant women, despite warnings from the healthcare centre midwife.

That I call the hospital and say: 'I'm sending you a woman for whom I just can't find foetal monitoring'. I call the hospital so that when she arrives, she doesn't experience 'What are you here for?' and the woman has to say, 'Look, it's just that my child died. ' (PCM7)

When the findings were brought back to the participants, one midwife suggested that this lack of communication is possibly due to structural deficiencies rather than a lack of communication between professionals.

Moreover, healthcare centres have the Connect 72 program, which is a program in which physicians and nurses automatically receive an alert for a telephone consultation within 72 hours after a patient is discharged. The system does not directly notify primary healthcare midwives, and although some cases are referred to midwives, not all are captured by them. Moreover, in early losses that did not require hospitalization, the system does not create the alert and the occurrence of a follow-up depends on whether the woman requests an appointment with the primary healthcare midwife. On the other hand, the call is made within 72 hours after discharge, which may be the time of greatest need for women.

Finding a motive to get involved

Despite the difficulties, midwives become more emotionally involved in the care when there is a strong bond with the mother, when they are better able to manage the involvement through coping strategies, when they feel a professional responsibility to care, and when they have also experienced a personal loss.

\section{Bonding with the mother}

The bond with the woman motivated midwives to go beyond task-focused care. This bond was created especially in primary healthcare. In many cases, women receive care from the same midwife during pregnancy, and a trusting relationship is built, in which women can express their feelings freely. This link was closer when losses were advanced, and therefore, the midwives' involvement was easier. 
From the point of view of midwives, the primary healthcare setting allowed them to relate to the experiences of the women and use the feedback from the women to reflect on and to improve the care.

In a healthcare centre, you create a bond with them, that they share things with you that they don't tell you in the hospital. Then it is when you realize 'Well, yes a miscarriage affects them'. (PCM9)

\section{Coping strategies}

The provision of care beyond medical tasks also depended on the coping tools of the midwives. Tools such as psychological therapy, which was used to enhance self-awareness of their feelings, helped them to understand the death in a way that allowed them to get more involved in the care. Many of the midwives understood IPLs as causes beyond them, as another possibility in life, or as unfavourable aspects of their work that could result in learning opportunities.

I personally went to therapy. I am very happy because I think it has helped me a lot to manage my issues, so as not be [negatively] affected by the issues of others. (PCM3)

Professional responsibility

Although the provision of care was a challenge for midwives, and it entailed personal costs to get involved in the care, midwives felt it was their responsibility as professionals. Previous situations in which they received negative feedback from the woman, motivated them to go beyond task-focused care. On the other hand, this implication in the care was strongly influenced by the midwives' personalities.

For me [the IPL] is one hundred percent my responsibility. And it wouldn't occur to me not to take care of her, even though it hurts. It hurts, but at the same time I want to take care of them. (PCM9)

Personal experiences with loss

Finally, personal losses motivate midwives to get more involved in caring, because they understood the couple's feelings and it was easier to empathize with them. One midwife reported the following: As a result of having abortions, you get a little more involved in ... And you can understand a little better what the other person is feeling. (PCM5)

Providing care from the rear

The care provide for these losses was based on the intuition of the midwives because of the lack of guidelines. Furthermore, the care of the father was an emerging concern in their narratives.

\section{Lack of guidelines}

Primary healthcare midwives do not have guidelines on providing care to parents who have experienced an IPL. Because of the lack of guidelines and knowledge, the care provided depends on the practices of each midwife and is based on their intuition. Furthermore, the healthcare follow-up of the woman is focused on her physical wellbeing, and consideration of other aspects depends on the requests of the woman.

So, it's to improvise, listen, and communicate. And sometimes, even, being there and saying, 'What do I do? What do I say?' Because I don't know. (PCM2)

The care is designed to allow the woman to express her experiences and feelings, listen to her, respect her time, resolve her doubts, and normalize her feelings. 
Providing care to the father

One aspect that emerged from the midwives' narratives was the care provided to the partner. Midwives commented that the healthcare is mainly focused on mothers, because of the physical aspects. Some midwives reported that they did not know how to empathize with the father or how to deal with the fathers' feelings. Two possible reasons for this may be the weakness of the prior bond between the father and the midwife and the social pressure on the father to be strong and inscrutable.

It is very difficult for men to open up, although I try to involve them. I don't know why, if it's because they don't have the confidence that women have with me or why. (PCM9)

\section{Avoiding emotional connections with the parents}

Lack of knowledge and time, personal costs, high emotional demands of getting involved in the care, and lack of effective coping strategies could hinder midwives from going beyond task-focused care. Midwives may feel that they are incapable of encountering the suffering of these parents. Therefore, midwives may avoid encounters with the woman and partner and engage in superficial communication with them. They may also experience fear of getting involved.

I think what we are trying to do is cover this up a bit. As we say, to pass soon, and sometimes what we have is fear of entering... Maybe not knowing what to do with the answers that women can say. (PCM8)

\section{Discussion}

This study focused on the meaning of lived experiences of primary healthcare midwives caring for parents who have suffered an IPL. For the midwives, this care involved handling adversity. Under these circumstances, they found motivation to go beyond task-focused care. In their provision of care the midwives followed their intuition or avoided encounters with the parents (figure 1).

Midwives may experience fear of being labelled unprofessional for providing caritative care or going beyond the medical task (Arman and Rehnsfeldt, 2006). Eriksson (2002) states that the basic motive of caring is the caritas motive, and, in the Western health systems, it means going beyond a role. Our findings showed ethos, or human value base, was what motivated midwives to get more involved in the care of these couples. This involvement is seen as a gift that the midwife gives the couples, as a result of a choice she makes because she wants to, not because she has to (Bolton, 2000). In this sense, the organizational culture could minimize the personal cost of this care by ensuring support for midwives and a caring culture, through training and the provision of spaces and time in which they can share their experiences and emotions.

According to the Caritative Caring Theory, genuine caring is provided to alleviate suffering and promote health and life (Eriksson, 1994). Midwives are essential professionals in the genuine care of IPLs because they are best positioned to build strong ties with the parents. This situation is facilitated in the case of primary healthcare midwives, because a previous relationship was surely established (Ellis et al., 2016; Gold, 2007). However, taking care of these parents is a challenge for them (Beaudoin and Ouellet, 2018; Jones and Smythe, 2015).

Dealing with death causes anxiety in nursing staff, and adequate knowledge about dying care is needed (Zomorodi and Lynn, 2010). According to Begley (2003), structured support during clinical experiences and education about bereavement and communication should be included in the training of midwives. However, in Western societies, death, particularly IPLs, are taboo, and it means the antithesis of the expected in the midwifery discipline (Granero-Moya et al., 2016; Lang et al., 2011).

The social context also has influence on the experiences of male partners. The literature highlights a societal pressure on men to 'be strong' and the belief that 'men don't share' (O'Leary and Thorwick, 2006; Samuelsson et al., 2001). However, IPLs have been shown to have an impact on them, but the manifestations of these feelings may be different from those of women. Lack of knowledge by healthcare professionals regarding men's experiences may lead to helplessness and marginalization (Due et al., 2017; 
McCreight, 2004). However, our results showed that what midwives did not know was how to approach them. This may be due to the lack of a previous tie and to the fact that the encounters with the midwife were carried out as a couple and the man wanted to keep the façade of strength in front of the woman. According to Strandås and Bondas (2018), the development and maintenance of a trusting relationship with the parents, specifically with the men, requires work on the part of midwives. They should be supported by the healthcare organizations, which should facilitate the establishment of a couple-midwife relationship. Moreover, further research is needed to shed light on the experiences of men in these losses and to promote the establishment of a trusting relationship.

Guidelines often focus on specialized care that involves the acute moment of loss, but follow-up is hardly addressed (Koopmans et al., 2013; Peters et al., 2016). Our results, in line with the literature, showed that the care provided by midwives was based on their intuition or their personal and professional experiences (Hutti et al., 2016; McCreight, 2004; Nash et al., 2018).

Follow-up is hindered by poor communication between specialized and primary healthcare. In our results, this aspect was controversial, because although it emerged from the analysis of the interviews, after participants' feedback, one midwife who previously reported this information, did not recognize the break between the two healthcare levels. This may be understood from the collaboration among professionals who are involved in the continuity of care. The involvement of other sectors is also necessary for continuity of care (Maarsingh et al., 2016). Various strategic collaboration plans have been carried out in our context, but they have not been successful in terms of continuity care (Guzmán-Fernández, 2011).

The literature highlights the important role of leaders in improving collaboration (Lemetti et al., 2015). According to Sjølie et al. (2020), first-line nursing managers are essential for improving the quality of care; they are able to change work practices, which leads to better outcomes for patients. However, in our context, the assistance units are physically far from the management ones (Guzmán-Fernández, 2011). It is necessary to have new forms of leadership and to professionalize management and strengthen competences and skills, including professionalism, leadership, decision-making, conflict management and problem-solving skills (Lemetti et al., 2015).

Our results highlighted the beneficial position of primary healthcare midwives in caring for these losses. The establishment of a previous emotional bond allowed the women to be more open and to share their experiences with the midwives. However, empathy and compassion may also entail additional emotional labour (McQueen, 2000). A market mentality and a predominant biomedical model can belittle the therapeutic value of that emotional involvement and promote the provision of task-focused care (McCreight, 2005; McQueen, 2000). This emotional demand can cause emotional exhaustion and professional burnout (Hunter and Smith, 2007; Huynh et al., 2008). In early losses, due to the high frequency in clinical practice, this exhaustion is often associated with the loss of the ability to provide compassionate care and a decrease in care quality (Houck, 2014).

\section{Strengths and limitations}

Narrative interview was an appropriate method for disclosing the meaning of midwives' lived experience. The different backgrounds of the research team enhanced the design and analysis phases of the study. Regarding the sample, this study provides the meaning of primary healthcare midwives' lived experiences, which offers a novel contribution not previously addressed in the body of knowledge. In addition, the sample was heterogeneous in relation to training and professional experience, which has enriched the knowledge of these experiences.

Results from this study can be transferred to other settings where midwives are present in primary healthcare. Moreover, the results may be beneficial for other professionals and disciplines with similar responsibilities to those performed by the midwives of this study.

Among the challenges of the study we highlight that the midwives were all women. According to them, being a woman might facilitate the establishment of a trusting bond with the mothers, with whom they felt comfortable to share their experience. 


\section{Conclusion}

The findings of this study showed that in caring for parents who have experienced an IPL, primary healthcare midwives leave their comfort zone. They experience high emotional demands and lack of knowledge and must engage in support and communication with specialized healthcare. The absence of care guidelines and the lack of recognition of primary healthcare in care recommendations can cause midwives to avoid encountering parents' suffering. However, they go beyond task-focused care, providing care based on their intuition, when they find motivation to get more involved.

Education and training are required in order to prepare midwives for encounters with parents who have suffered an IPL. Leadership is also needed to facilitate care continuity through the improvement of communication between specialized and primary healthcare and the promotion of the welfare of midwives. This study fills a gap by elucidating the meaning of primary healthcare midwives' lived experiences, and can bring about change in clinical practice, by informing improvement efforts in the care of parents who suffer IPLs.

\section{Ethical approval}

The study obtained the approval of the Autonomous Committee of Research Ethics of Galicia (registration code 2015/232) and had the access permission by health area. All participants provided written informed consent to participate in the study.

\section{Funding sources}

This work was supported by the Xunta de Galicia [Orde 29/05/2017 (Dog. $\mathrm{n}^{\circ} 109$ (Friday, June 9th 2017)].

\section{CRediT authorship contribution statement}

Sara Fernández-Basanta: Conceptualization, Methodology, Formal analysis, Investigation, Resources, Writing - original draft, Writing - review \& editing, Project administration, Funding acquisition. Carmen Coronado: Conceptualization, Methodology, Formal analysis, Resources, Writing - review \& editing, Supervision, Project administration, Funding acquisition. Terese Bondas: Methodology, Resources, Writing - review \& editing, Supervision. María-Jesús Movilla-Fernández: Conceptualization, Methodology, Formal analysis, Resources, Writing - review \& editing, Supervision, Project administration, Funding acquisition.

\section{Declaration of competing interest}

The authors state that there is no conflict of interest.

\section{Acknowledgements}

The authors thank the participation of the primary healthcare midwifes in the study for their generosity in sharing their experience. They also express their gratitude to Healthcare Area Management of Ferrol for its readiness and willingness for this study to be carried out. 


\section{References}

Arman, M., Rehnsfeldt, A., 2006. The Presence of Love in Ethical Caring. Nurs. Forum 41 (1), 4-12. doi:10.1111/j.1744-6198.2006.00031.x.

Beaudoin, M.A., Ouellet, N., 2018. An exploration of factors influencing nursing practice with families experiencing perinatal loss. Rech. Soins. Infirm (133) 58-69. doi:10.3917/rsi.133.0058.

Begley, C., 2003. 'I cried... I had to...': student midwives' experiences of stillbirth, miscarriage and neonatal death. Evidence-Based Midwifery 1 (1), 20-27.

Bolton, S.C., 2000. Who cares? Offering emotion work as a 'gift' in the nursing labour process. J. Adv. Nurs 32 (3), 580-586. doi:10.1046/j.1365-2648.2000.01516.x.

Due, C., Chiarolli, S., Riggs, D.W., 2017. The impact of pregnancy loss on men's health and wellbeing: a systematic review. BMC Pregnancy Childbirth 17 (1), 380. doi:10.1186/s12884-017-1560-9.

Ellis, A., Chebsey, C., Storey, C., Bradley, S., Jackson, S., Flenady, V., Heazell, A., Siassakos, D., 2016. Systematic review to understand and improve care after stillbirth: a review of parents' and healthcare professionals' experiences. BMC Pregnancy Childbirth 16, 16. doi:10.1186/s12884-016-0806-2.

Eriksson, K., 1994. Theories of caring as health. In: Gaut, D.A., Boykin, A. (Eds.), Caring as healing: Renewal through hope. National League for Nursing, New York, pp. 2-20.

Eriksson, K., 2002. Caring science in a new key. Nurs. Sci. Q 15 (1), 61-65. doi:10.1177/089431840201500110.

Fernández-Basanta, S., Coronado, C., Movilla-Fernández, M. J., 2020a. Multicultural coping experiences of parents following perinatal loss: A meta-ethnographic synthesis. J. Adv. Nurs. 76 (1), 9-21.

Fernández-Basanta, S., Movilla-Fernández, M.J., Coronado, C., Llorente-García, H., Bondas, T., 2020b. Involuntary pregnancy loss and nursing care: a meta-ethnography. Int. J. Environ. Res. Public Health 17 (5), 1486

Fernández-Basanta, S., Van, P., Coronado, C., Torres, M., Movilla-Fernández, M.J., 2019. Coping after involuntary pregnancy loss: Perspectives of Spanish European women. OMEGA-J. Death Dying doi:10.1177/0030222819852849.

Flenady, V., Boyle, F., Koopmans, L., Wilson, T., Stones, W., Cacciatore, J., 2014. Meeting the needs of parents after a stillbirth or neonatal death. BJOG 121 (s4), 137-140. doi:10.1111/1471-0528.13009.

Gold, K., 2007. Navigating care after a baby dies: a systematic review of parent experiences with health providers. J Perinatol 27 (4), 230-237. doi:10.1038/sj.jp.7211676.

Granero-Moya, N., Frías-Osuna, A., Barrio-Cantalejo, I.M., Ramos-Morcillo, A.J., 2016. Dificultades de las enfermeras de atención primaria en los procesos de planificación anticipada de las decisiones: un estudio cualitativo. Aten Primaria 48 (10), 649-656. doi:10.1016/j.aprim.2016.01.008.

Guzmán-Fernández, M.Á., 2011. Pérdida de poder de las direcciones de enfermería de Atención Primaria. Enferm Clin 21 (4), 187-188.

Heazell, A.E., Wojcieszek, A., Graham, N., Stephens, L., 2019. Care in pregnancies after stillbirth and perinatal death. Int. J. Birth Parent Educ 6 (2), 23-28.

Houck, D., 2014. Helping nurses cope with grief and compassion fatigue: an educational intervention. Clin J Oncol Nurs 18 (4).

Hunter, B., Smith, P., 2007. Emotional labour: Just another buzz word? Int J Nurs Stud 44 (6), 859-861. doi:10.1016/j.ijnurstu.2006.12.015.

Hutti, M.H., Polivka, B., White, S., Hill, J., Clark, P., Cooke, C., Clemens, S., Abell, H., 2016. Experiences of Nurses Who Care for Women After Fetal Loss. J Obstet Gynecol Neonatal Nurs 45 (1), 17-27. doi:10.1016/j.jogn.2015.10.010.

Huynh, T., Alderson, M., Thompson, M., 2008. Emotional labour underlying caring: an evolutionary concept analysis. J. Adv. Nurs 64 (2), 195-208. doi:10.1111/j.1365-2648.2008.04780.x.

Jones, K., Smythe, L., 2015. The impact on midwives of their first stillbirth. N. Z. Coll. Midwives J. (51) 1722. doi:10.12784/nzcomjn151.2015.3.17-22.

Koopmans, L., Wilson, T., Cacciatore, J., Flenady, V., 2013. Support for mothers, fathers and families after perinatal death. Cochrane Database Syst Rev (6), CD000452 doi:10.1002/14651.858.CD000.452.pub3.

Lang, A., Fleiszer, A.R., Duhamel, F., Sword, W., Gilbert, K.R., Corsini-Munt, S., 2011. Perinatal loss and parental grief: the challenge of ambiguity and disenfranchised grief. Omega 63 (2), 183-196. doi:10.2190/OM.63.2.e.

Lemetti, T., Stolt, M., Rickard, N., Suhonen, R., 2015. Collaboration between hospital and primary care nurses: a literature review. Int Nurs Rev 62 (2), 248-266. doi:10.1111/inr.12147.

Lindseth, A., Norberg, A., 2004. A phenomenological hermeneutical method for researching lived experience. Scand J Caring Sci 18 (2), 145-153. doi:10.1111/j.1471-6712.2004.00258.x.

López García de Madinabeitia, A.P., 2011. Duelo perinatal: un secreto dentro de un misterio. Rev. Asoc. Esp. Neuropsiquiatr 31 (1), 53-70. doi:10.4321/S0211-57352.01100.0100005.

Maarsingh, O.R., Henry, Y., van de Ven, P.M., Deeg, D.J., 2016. Continuity of care in primary care and association with survival in older people: a 17-year prospective cohort study. Br J Gen Pract 66 (649), e531-e539. doi:10.3399/bjgp16X686101. 
Martínez-Serrano, P., Palmar-Santos, A.M., Solis-Munoz, M., Alvarez-Plaza, C., PedrazMarcos, A., 2018. Midwives' experience of delivery care in late foetal death: A qualitative study. Midwifery 66, 127-133. doi:10.1016/j.midw.2018.08.010.

McCreight, B.S., 2004. A grief ignored: narratives of pregnancy loss from a male perspective. Sociol Health Illn 26 (3), 326-350. doi:10.1111/j.1467-9566.2004.00393.x.

McCreight, B.S., 2005. Perinatal grief and emotional labour: a study of nurses' experiences in gynae wards. Int J Nurs Stud 42 (4), 439-448. doi:10.1016/j.ijnurstu.2004.07.004.

McQueen, A., 2000. Nurse-patient relationships and partnership in hospital care. J Clin Nurs 9 (5), 723-731. doi:10.1046/j.1365-2702.2000.00424.x.

Nash, M., Barry, M., Bradshaw, C., 2018. Midwives' experiences of caring for women with early pregnancy loss in an Irish maternity hospital. British Journal of Midwifery 26 (12), 796-805. doi:10.12968/bjom.2018.26.12.796.

O'Leary, J., Thorwick, C., 2006. Fathers' perspectives during pregnancy, postperinatal loss. J Obstet Gynecol Neonatal Nurs 35 (1), 78-86. doi:10.1111/j.1552-6909.2006.00017.x.

Peters, M.D., Lisy, K., Riitano, D., Jordan, Z., Aromataris, E., 2016. Providing meaningful care for families experiencing stillbirth: a meta-synthesis of qualitative evidence. J Perinatol 36 (1), 3-9. doi:10.1038/jp.2015.97.

Ricoeur, P., 1976. Interpretation theory: Discourse and the surplus of meaning. Texas Christian University Press.

Samuelsson, M., Rådestad, I., Segesten, K., 2001. A waste of life: fathers' experience of losing a child before birth. Birth 28 (2), 124-130. doi:10.1046/j.1523-536X.2001.00124.x.

Sjølie, B.M., Hartviksen, T.A., Bondas, T., 2020. "Navigation to prioritizing the patient"-first-line nurse managers' experiences of participating in a quality improvement collaborative. BMC Health Serv Res 20 (1), 55. doi:10.1186/s12913-020-4918-z.

Strandås, M., Bondas, T., 2018. The nurse-patient relationship as a story of health enhancement in community care: A meta-ethnography. J. Adv. Nurs 74 (1), 11-22. doi:10.1111/jan.13389.

Wiklund, L., Lindholm, L., Lindström, U.A., 2002. Hermeneutics and narration: a way to deal with qualitative data. Nurs Inq 9 (2), 114-125.

Zomorodi, M., Lynn, M.R., 2010. Critical care nurses' values and behaviors with end-of-life care: perceptions and challenges. J Hosp Palliat Nurs 12 (2), 89-96. doi:10.1097/NJH.0b013e3181cf7cf6. 